\title{
Thermal transport properties of decagonal quasicrystals and their approximants
}

\author{
Petar Popčević ${ }^{1}$, Ante Bilušić ${ }^{1,2}$, Kristijan Velebit ${ }^{1}$ and Ana Smontara ${ }^{1}$ \\ ${ }^{1}$ Institut za fiziku, Bijenička 46, 10000 Zagreb, Croatia \\ ${ }^{2}$ Faculty of Science, University of Split, Nikole Tesle 12, 21000 Split, Croatia
}

\begin{abstract}
Transport properties (thermal conductivity, electrical resistivity and thermopower) of decagonal quasicrystal $d$ - $\mathrm{AlCoNi}$, and approximant phases $\mathrm{Y}-\mathrm{AlCoNi}, \mathrm{o}-\mathrm{Al}_{13} \mathrm{Co}_{4}, \mathrm{~m}-\mathrm{Al}_{13} \mathrm{Fe}_{4}, \mathrm{~m}$ $\mathrm{Al}_{13}(\mathrm{Fe}, \mathrm{Ni})_{4}$ and $\mathrm{T}-\mathrm{AlMnFe}$ have been reviewed. Among all presented alloys the stacking direction (periodic for decagonal quasicrystals) is the most conductive one for the charge and heat transport, and the in/out-of-plane anisotropy is much larger than the in-plane anisotropy. There is a strong relationship between periodicity length along stacking direction and anisotropy of transport properties in both quasicrystals and their approximants suggesting a decrease of the anisotropy with increasing number of stacking layers.
\end{abstract}

\section{INTRODUCTION}

Since their discovery in 1982 by D. Shechtman [1], quasicrystals attract great attention due to their unique atomic structure and very interesting physical properties that result from it. They are mostly ternary alloys usually built on the basis of aluminum and transition metals. According to their crystal structure we distinguish two classes of quasicrystals: icosahedral and polygonal quasicrystals. In icosahedral quasicrystals [2], atoms are packed in clusters with icosahedral symmetry possessing quasiperiodic ordering along all three spatial directions. Among polygonal quasicrystals, we distinguish octagonal [3], decagonal [4] and dodecagonal [5] quasicrystals possessing quasiperiodic eightfold, tenfold and twelvefold symmetry axes, respectively, while they are periodically ordered along these symmetry axes. A possibility to grow crystals of decagonal phase of exceptional quality [6] makes them a good candidate for studying physical distinctions of periodic and quasiperiodic orderings on the same specimen. Besides that, a class of complex metallic alloys called approximants to the decagonal quasicrystals proved to be very useful in the task. Approximant phases are alloys possessing local atomic arrangements very similar to those in quasicrystals but retaining periodicity, which makes them an ordinary subject of theoretical investigation. It is well known that the degree of anisotropy of the transport properties of polygonal quasicrystals depends on the number of the quasiperiodic layers along one periodic unit [7]. Prior measurements of the electrical resistivity, thermopower [8] and thermal conductivity [9] have provided the motivation to thoroughly investigate the composition dependent transport properties (electrical resistivity $(\rho)$, thermopower (S) and thermal conductivity $(\kappa)$ ) of decagonal quasicrystals and their approximants.

\section{EXPERIMENT}

In this study we investigated several approximant phases to the decagonal quasicrystals. Their structure can be viewed as stacking of pseudotenfold layers along the direction that corresponds to the tenfold direction in decagonal phase. We have studied $\mathrm{Y}-\mathrm{Al}-\mathrm{Co}-\mathrm{Ni}$ [10] phase with two stacking layers, o- $\mathrm{Al}_{13} \mathrm{Co}_{4}$ [7], $\mathrm{m}-\mathrm{Al}_{13} \mathrm{Fe}_{4}$ and its ternary extension m- $\mathrm{Al}_{13}(\mathrm{Fe}, \mathrm{Ni})_{4}[11]$ 
with four stacking layers and Taylor phase T-Al-Mn-Fe [12] with six stacking layers. Finally we correlated their transport properties to those of decagonal $d-\mathrm{Al}_{70} \mathrm{Co}_{10} \mathrm{Ni}_{20}$ [13] with two stacking layers.

In order to perform crystallographic-direction-dependent studies, we have cut from the single crystal of each compound three bar-shaped samples of dimensions $1 \times 1 \times 8 \mathrm{~mm}^{3}$, with their long edges along three orthogonal directions.

The electrical resistivity and thermal conductivity were measured in a temperature interval from 1.5 to $300 \mathrm{~K}$ using the self-made sample holders, with a standard four point method and an absolute steady state method, respectively. The thermopower was measured using a standard temperature gradient technique [14] employing $50 \mathrm{~mm}$ thick $\mathrm{Au}(0.07$ at. \% Fe)-Chromel thermocouple. In the thermal conductivity measurements the sample was glued to the heat sink on one side, while the $\mathrm{RuO}_{2}$ resistor was attached to the other side in order to maintain the temperature gradient across the sample [15]. The gradient was monitored with $25 \mathrm{~mm}$ thick $\mathrm{Au}(0.07$ at. $\% \mathrm{Fe})$-Chromel thermocouple.

\section{RESULTS AND DISCUSSION}

The electrical resistivities of three approximant phases having two, four and six stacking layers are shown in figure 1. Y-AlCoNi (two stacking layers) has the lowest electrical resistivity, which at room temperature amounts to $25 \mu \Omega \mathrm{cm}$ along the stacking direction and $60 \mu \Omega \mathrm{cm}$ and $80 \mu \Omega \mathrm{cm}$ perpendicular to the stacking direction with the anisotropy of 3.2. Resistivity of o$\mathrm{Al}_{13} \mathrm{Co}_{4} \mathrm{Al}_{13} \mathrm{Co}_{4}$ (four stacking layers) at room temperature amounts to $70 \mu \Omega \mathrm{cm}$ along the stacking direction and $170 \mu \Omega \mathrm{cm}$ and $180 \mu \Omega \mathrm{cm}$ perpendicular to the stacking direction with the anisotropy of 2.6. While those two alloys show metallic positive temperature coefficient of the electrical resistivity, T-AlMnFe with six stacking layers shows negative temperature coefficient with the room temperature value of the electrical resistivity of $440 \mu \Omega \mathrm{cm}$ along the stacking direction and $490 \mu \Omega \mathrm{cm}$ and $500 \mu \Omega \mathrm{cm}$ perpendicular to the stacking direction with a minor anisotropy of 1.2 .
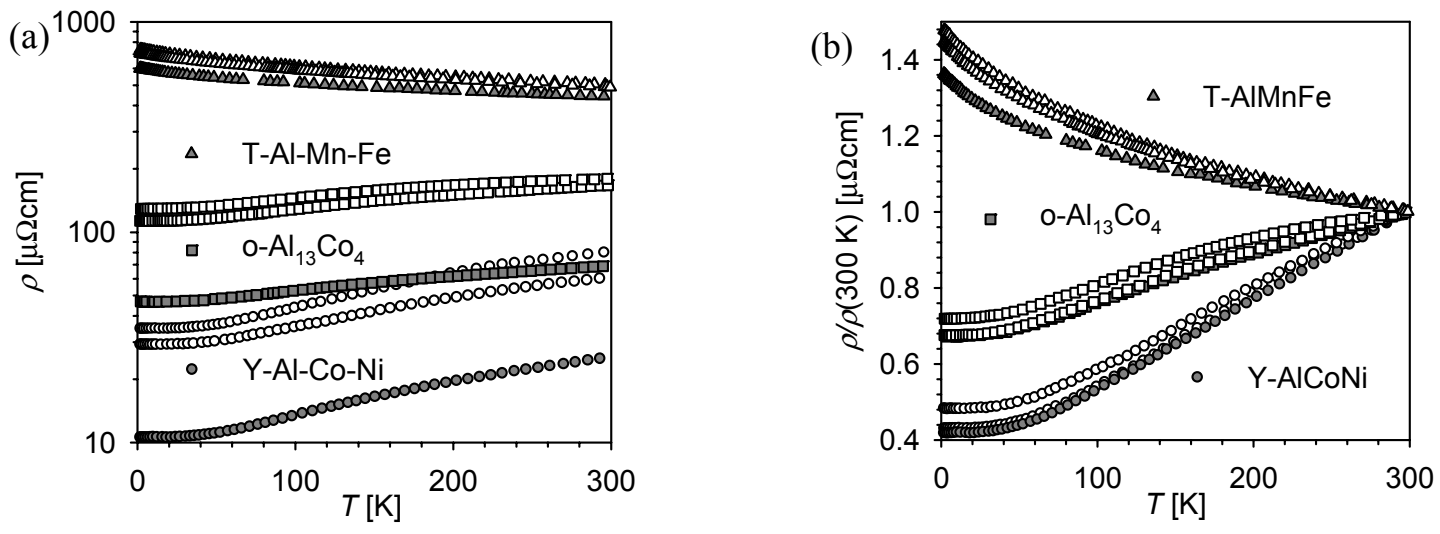

Figure 1. (a) Electrical resistivity and (b) electrical resistivity scaled to the room temperature value of Y$\mathrm{AlCoNi}, \mathrm{o}-\mathrm{Al}_{13} \mathrm{Co}_{4}$ and $\mathrm{T}-\mathrm{AlMnFe}$. Gray and open symbols denote electrical resistivity along and perpendicular to the stacking direction respectively [16].

Figure 1 thus leads us to conclude that the electrical resistivity increases and anisotropy decreases as the number of stacking layers increase. However, this is not completely true, since 
monoclinic phase $\mathrm{m}-\mathrm{Al}_{13} \mathrm{Fe}_{4}$ with four stacking layers has an anisotropy higher than $\mathrm{Y}-\mathrm{AlCoNi}$, but it also has a significant inlayer anisotropy and more pronounced temperature dependence (figure 2).
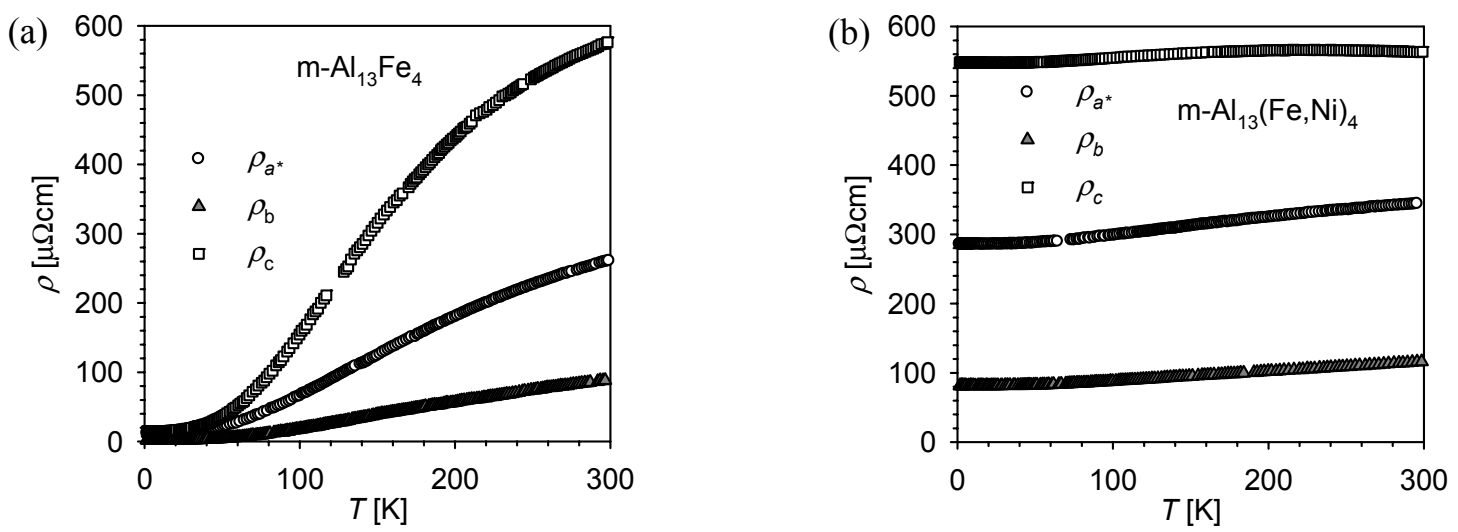

Figure 2. Electrical resistivity of (a) $\mathrm{m}-\mathrm{Al}_{13} \mathrm{Fe}_{4}$ and (b) its ternary extension $\mathrm{m}-\mathrm{Al}{ }_{13}(\mathrm{Fe}, \mathrm{Ni})_{4}$ with four stacking layers [11].

Comparing the electrical resistivity of $\mathrm{m}-\mathrm{Al}_{13} \mathrm{Fe}_{4}$ with the one of its ternary extension m$\mathrm{Al}_{13}(\mathrm{Fe}, \mathrm{Ni})_{4}$, it can be concluded that chemical and structural disorder, that is characteristic for the complex metallic alloys, plays an important role in determining their transport properties.

The thermal conductivities of $\mathrm{Y}-\mathrm{AlCoNi}, \mathrm{O}-\mathrm{Al}_{13} \mathrm{Co}_{4}$ and $\mathrm{T}-\mathrm{AlMnFe}$ are presented in figure 3. The thermal conductivity is highest in the $\mathrm{Y}-\mathrm{AlCoNi}$ with the value of $46.3 \mathrm{~W} / \mathrm{mK}$ at room temperature along the stacking direction and $12.5 \mathrm{~W} / \mathrm{mK}$ and $17.4 \mathrm{~W} / \mathrm{mK}$ perpendicular to the stacking direction with the anisotropy of 3 . In o- $\mathrm{Al}_{13} \mathrm{Co}_{4}$ the room-temperature thermal conductivity along the stacking direction amounts to $12.5 \mathrm{~W} / \mathrm{mK}$, while perpendicular to the stacking direction it amounts to $6.1 \mathrm{~W} / \mathrm{mK}$ and $6.2 \mathrm{~W} / \mathrm{mK}$ with the anisotropy of 2 . Taylor phase $\mathrm{T}$-AlMnFe shows no significant anisotropy in thermal transport with the thermal conductivity along all three crystallographic axes amounting to $3.5 \mathrm{~W} / \mathrm{mK}$ at $300 \mathrm{~K}$ within the experimental error.
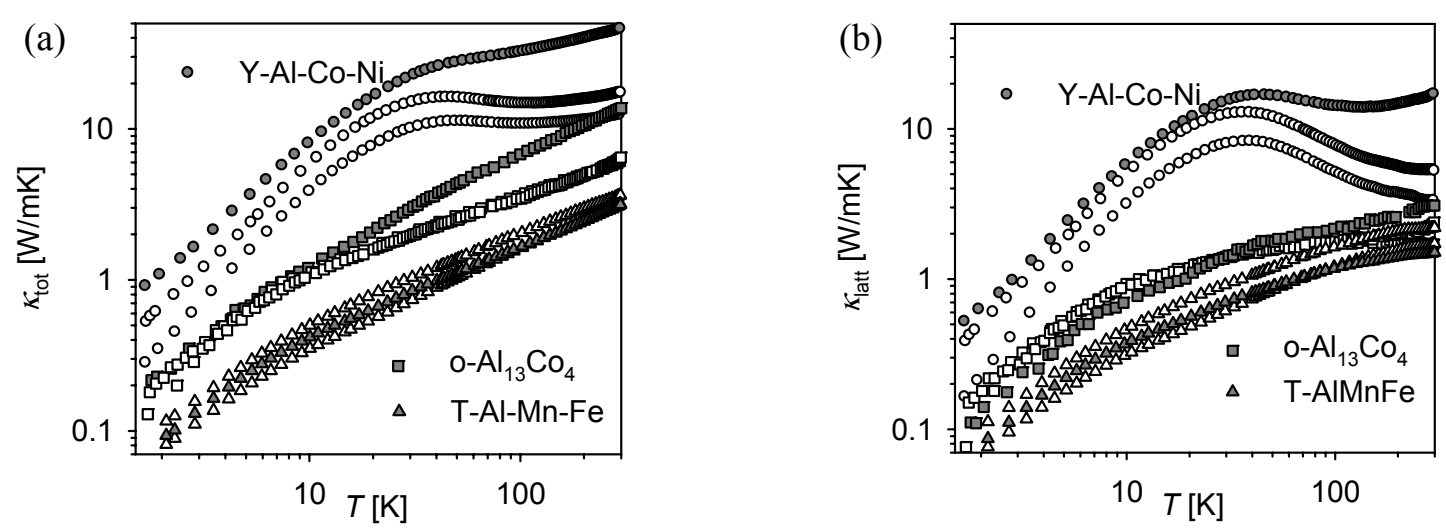

Figure 3. (a) Total and (b) lattice thermal conductivity derived using Wiedemann-Franz Law of Y$\mathrm{AlCoNi}, \mathrm{o}-\mathrm{Al}_{13} \mathrm{Co}_{4}$ and $\mathrm{T}-\mathrm{AlMnFe}$. Gray and open symbols denote thermal conductivity along and perpendicular to the stacking direction respectively. 
The lattice contribution to the thermal conductivity $\left(\kappa_{\text {lat }}\right)$ was derived by subtracting the electroxnic contribution to the thermal conductivity $\left(\kappa_{\mathrm{el}}\right)$ from the total thermal conductivity $\left(\kappa_{\text {tot }}\right)$. The $\kappa_{\mathrm{el}}$ was estimated using Wiedemann-Franz law. Although WF law is valid only when the electronic scattering is elastic (or quasielastic at temperatures above Debye temperature) [17], we use it as a rough estimate, since the temperature dependence of the electrical resistivity is not large, suggesting a significant proportion of scattering is due to the elastic scattering on crystal defects rather than the inelastic ones on phonons.
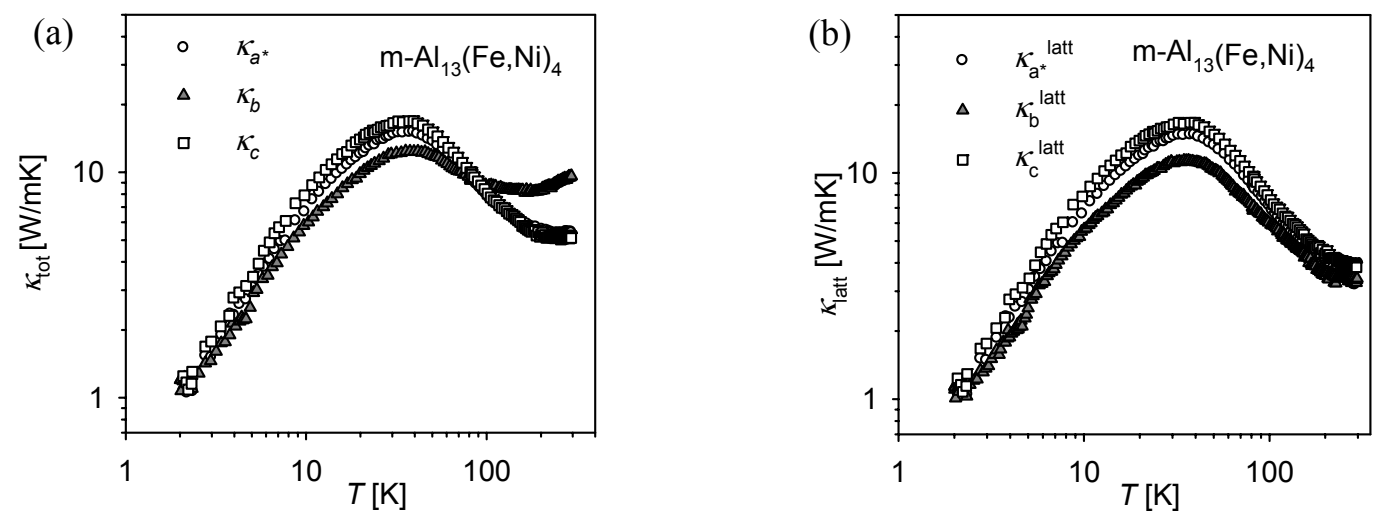

Figure 4. (a) Total and (b) lattice thermal conductivity derived using Wiedemann-Franz Law of m$\mathrm{Al}_{13}(\mathrm{Fe}, \mathrm{Ni})_{4}$. Gray and open symbols denote thermal conductivity along and perpendicular to the stacking direction respectively.

The lattice contribution to the thermal conductivity shows smaller anisotropy than $\kappa_{\text {tot }}$, and it does not differ much among different alloys. Furthermore, if we look at the thermal conductivity of $\mathrm{m}-\mathrm{Al}_{13}(\mathrm{Fe}, \mathrm{Ni})_{4}$ (figure 4$)$, regardless of the presence of the large umklapp maximum, the thermal conductivity at the room temperature is almost the same as in o- $\mathrm{Al}_{13} \mathrm{Co}_{4}$, and $\kappa_{\text {latt }}$ shows no anisotropy at the room temperature. Faster saturation of the anisotropy of the $\kappa_{\text {latt }}$ with increasing of the complexity of crystal structure suggests that phonons are more affected by it, while the electrical resistivity is more sensitive to the structure details resulting in larger anisotropy.
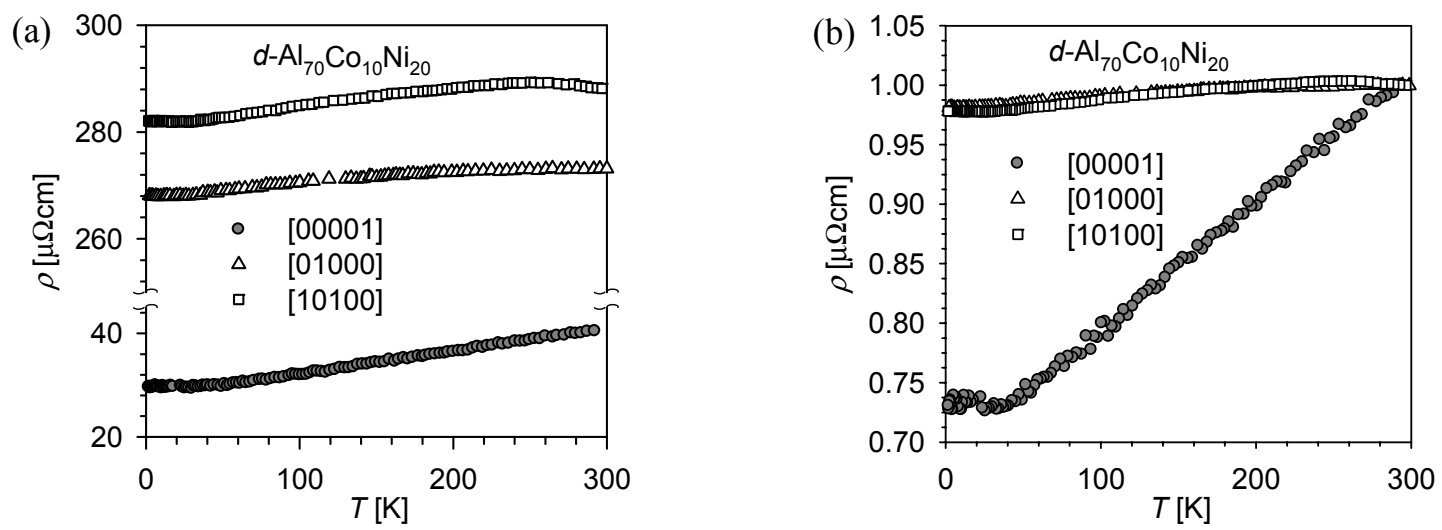

Figure 5. (a) Electrical resistivity of decagonal $d$-AlCoNi quasicrystal. (b) Electrical resistivity of $d$ AlCoNi normalized at the room temperature value. 
The electrical resistivity of $d$-AlCoNi presented in figure 5 is in good agreement with the literature [18]. At the room temperature it amounts to $40 \mu \Omega \mathrm{cm}$ along stacking direction, while in the quasiperiodic directions it amounts to $274 \mu \Omega \mathrm{cm}$ and $288 \mu \Omega \mathrm{cm}$ with the anisotropy of 6 . Contrary to the approximant phases, a significant anisotropy in the decagonal phase is obtained in the temperature coefficient of the electrical resistivity: the residual resistivity ratio along the stacking direction is 1.33 , while in the quasiperiodic directions it is only 1.02. This anisotropy is related to the existence of periodicity along the stacking direction which is absent in the quasiperiodic plane.
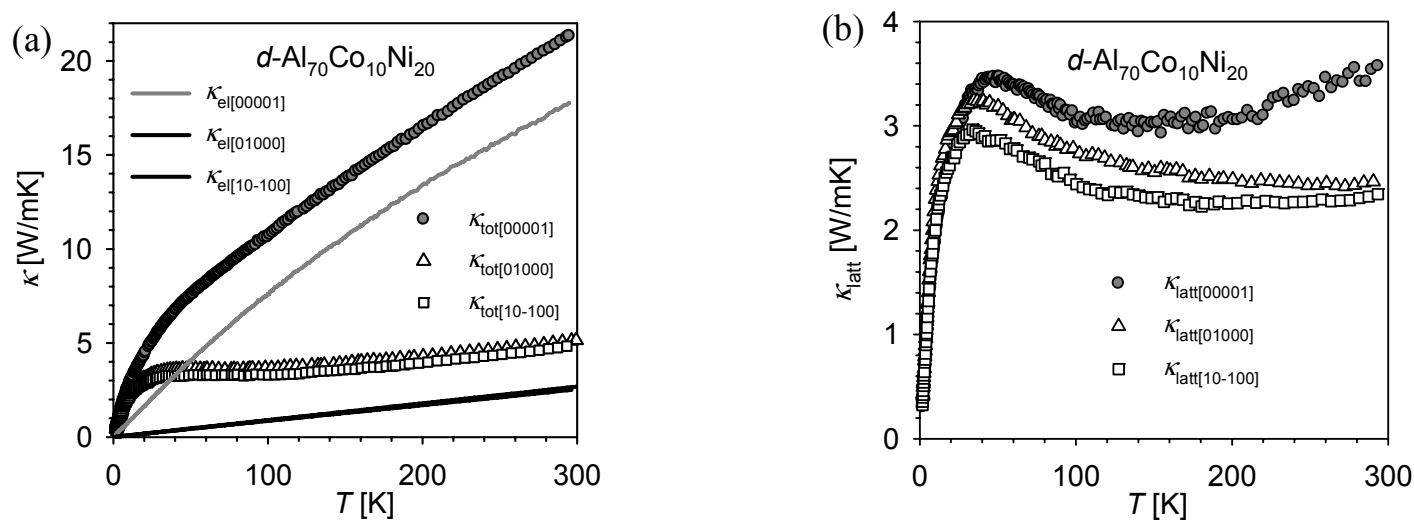

Figure 6. (a) Total thermal conductivity and electronic contribution to the thermal conductivity estimated using Wiedeman-Franz law, and (b) Lattice contribution to the thermal conductivity of decagonal $d$ $\mathrm{Al}_{70} \mathrm{Co}_{10} \mathrm{Ni}_{20}$ quasicrystal.

The thermal conductivity of the $d$-AlCoNi presented in figure 6 , is very similar to the one reported previously [19] on the $d-\mathrm{Al}_{70} \mathrm{Co}_{15} \mathrm{Ni}_{15}$. At the room temperature, the thermal conductivity amounts to $21 \mathrm{~W} / \mathrm{mK}$ along the stacking direction, while in quasiperiodic plane it amounts to 4.8 and $5 \mathrm{~W} / \mathrm{mK}$ with the anisotropy of 4.3 . Despite such large anisotropy of $\kappa_{\text {tot }}, \kappa_{\text {latt }}$ (figure 6 (b)) shows almost no anisotropy demonstrating that anisotropy principally arises from electronic system of $d$-AlCoNi. Contrary to the reported thermal conductivity [19], there is saturation in $\kappa_{\text {latt }}$ along quasiperiodic directions at the room temperature, while along periodic direction an upturn is observed that can be attributed to the hopping of the localized vibrations [20].

\section{CONCLUSIONS}

We have investigated series of approximants to the decagonal quasicrystals with two, four and six stacking layers. We conclude that the number of stacking layers is not the most important parameter that influences their transport properties rather is closely related to the size of the unit cell, and thus plays a role in $l / a$ ratio ( $l$ - electron mean free path, $a$ - size of unit cell) which is important for the magnitude of the temperature variation of the electrical resistivity. Other factor is the degree of the structural and chemical disorder that is characteristic for the complex metallic alloys, and great attention has to be placed on the crystal preparation.

In decagonal $d-\mathrm{Al}_{70} \mathrm{Co}_{10} \mathrm{Ni}_{20}$ quasicrystal, the main contribution to the anisotropy of transport properties comes from the electronic system, while it seems that phonons see mainly 
isotropic medium which is rather unexpected, but in accordance with the study of the elastic moduli of the same phase [21].

\section{ACKNOWLEDGMENTS}

The authors are grateful to P. Gille (Ludwig-Maximilians-Universität. München) and M. Feuerbacher and M. Heggen (Institut für Festkörperforschung, Forschungszentrum Jülich) for providing us with the high-quality samples. Many thanks to J. Dolinšek for valuable discussions. We acknowledge support by the MSES of the Republic of Croatia through Project No. 0350352826-2847.

\section{REFERENCES}

1. D. Shechtman, I. Blech, D. Gratias and J. W. Cahn, Phys. Rev. Lett. 53, 1951 (1984).

2. A. P. Tsai, J. Q. Guo, E. Abe, H. Takakura and T. J. Sato, Nature 408, 537 (2000).

3. W. Cao, H. Q. Ye and K. H. Kuo, Phys. Status Solidi A 107, 511 (1988).

4. W. Steurer, Z. Kristallogr. 219, 391 (2004).

5. H. Chen, D. X. Li and K. H. Kuo, Phys. Rev. Lett. 60, 1645 (1988).

6. P. Gille, P. Dreier, M. Gräber and T. Scholpp, J. Cryst. Growth 207, 95 (1999).

7. J. Dolinšek, M. Komelj, P. Jeglič, S. Vrtnik, D. Stanić, P. Popčević, J. Ivkov, A. Smontara, Z. Jagličić, P. Gille and Y. Grin, Phys. Rev. B 79, 184201 (2009).

8. S.-Y. Lin, X.-M. Wang, L. Lu, D.-L. Zhang, L. X. He and K. X. Kuo, Phys. Rev. B 41, 9625 (1990).

9. D.-L. Zhang, S.-C. Cao, Y.-P. Wang, L. Lu, X.-M. Wang, X. L. Ma and K. H. Kuo, Phys. Rev. Lett. 66, 2778 (1991).

10. A. Smontara, I. Smiljanić, J. Ivkov, D. Stanić, O. S. Barišić, Z. Jagličić, P. Gille, M. Komelj, P. Jeglič, M. Bobnar and J. Dolinšek, Phys. Rev. B 78, 104204 (2008).

11. P. Popčević, A. Smontara, J. Ivkov, M. Wencka, M. Komelj, P. Jeglič, S. Vrtnik, M. Bobnar, Z. Jagličić, B. Bauer, P. Gille, H. Borrmann, U. Burkhardt, Y. Grin and J. Dolinšek, Phys. Rev. B 81, 184203 (2010).

12. M. Heggen, M. Feuerbacher, J. Ivkov, P. Popčević, I. Batistić, A. Smontara, M. Jagodič, Z. Jagličić, J. Janovec, M. Wencka and J. Dolinšek, Phys. Rev. B 81, 184204 (2010).

13. M. Bobnar, P. Jeglič, M. Klanjšek, Z. Jagličić, M. Wencka, P. Popčević, J. Ivkov, D. Stanić, A. Smontara, P. Gille and J. Dolinšek, Phys. Rev. B 85, 024205 (2012).

14. A. Smontara, K. Biljakovic, J. Mazuer, P. Monceau and F. Levy, J. Phys. Condens. Matter 4, 3273 (1992).

15. A. Smontara, A. Bilušić, Ž. Bihar and I. Smiljanić, in Properties And Applications of Complex Intermetallics, edited by E. Belin-Ferré (World Scientific, 2009), pp. 113.

16. A. Smontara, P. Popčević, D. Stanić, K. Velebit and J. Dolinšek, Philos. Mag. 91, 2746 (2010).

17. J. M. Ziman, Electrons and Phonons: The Theory of Transport Phenomena in Solids. (Oxford University Press, London, 1960), pp. 260.

18. J. T. Markert, J. L. Cobb, W. D. Bruton, A. K. Bhatnagar, D. G. Naugle and A. R. Kortan, J. Appl. Phys. 76, 6110 (1994).

19. A. D. Bianchi, F. Bommeli, E. Felder, M. Kenzelmann, M. A. Chernikov, L. Degiorgi, H. R. Ott and K. Edagawa, Phys. Rev. B 58, 3046 (1998).

20. P. Popčević, D. Stanić ,Ž. Bihar , A. Bilušić and A. Smontara, Isr. J. Chem. 51, 1340 (2011). 
21. M. A. Chernikov, H. R. Ott, A. Bianchi, A. Migliori and T. W. Darling, Phys. Rev. Lett. 80, 321 (1998). 